\title{
Transdiagnostic CBT for adult emotional disorders: A feasibility open trial from Saudi Arabia
}

\author{
Yousra Alatiq* and Omar Al Modayfer \\ Mental Health Division, Department of Medicine, King Abdulaziz Medical City-Riyadh (KAMC-R) and King Saud bin \\ Abdulaziz University for Health Sciences (KSAU-HS), KSA \\ *Corresponding author. Emails: yalatiq@gmail.com; atiqy@ngha.med.sa
}

(Received 29 August 2018; revised 21 February 2019; accepted 25 March 2019)

\begin{abstract}
Transdiagnostic cognitive behavioural therapy (T-CBT) provides potential for improving psychotherapy services in countries with limited resources. The primary aim of this study was to assess the feasibility and potential benefits of using T-CBT in Saudi Arabia to treat adult emotional disorders in a naturalistic open trial. A secondary aim was to measure the effect of this approach when delivered by junior psychologists as a low-intensity intervention. The overall sample consisted of 198 patients (160 in the low-intensity group). Only $33(16.7 \%)$ patients had completed the treatment plan, 55 (27.3\%) were still active in treatment, and 109 (55\%) had disengaged from the treatment. The pre- and post-assessments for the clients who completed the treatment showed a significant decrease in all outcome measures. This result held true for the whole sample and the low-intensity group. This study provides initial evidence that T-CBT is suitable for clients with emotional disorders in Saudi Arabia. The study also provides support for the effect of T-CBT as a low-intensity intervention delivered by junior psychologists. However, one of the study limitations was the sample size for the group who completed the treatment and was properly discharged from service. Implications and recommendations are discussed.
\end{abstract}

\section{Key learning aims}

(1) To examine the feasibility and potential benefits of using T-CBT in Saudi Arabia.

(2) To measure the effect of T-CBT as low-intensity interventions delivered by junior psychologists.

(3) To establish evidence-based practice for T-CBT in Saudi Arabia.

Keywords: CBT; emotional disorder; low intensity; Saudi Arabia; transdiagnostic

\section{Introduction}

Transdiagnostic cognitive behavioural therapy (T-CBT) can be used to treat a number of different disorders that share common underlying cognitive, behavioural and/or emotional processes (Harvey et al., 2004). T-CBT was first introduced by Fairburn et al. (2003) to treat different eating disorders, then interest moved to anxiety and mood disorders (Barlow et al., 2004). Currently, a number of T-CBT protocols have been developed by different research groups (e.g. Barlow et al., 2011; Norton, 2012a). The effectiveness of such protocols has been examined in a number of meta-analyses and systematic reviews, and the overall findings suggest that T-CBT is effective in the treatment of emotional disorders (García-Escalera et al., 2017) when compared with control conditions (Andersen et al., 2016) and is as effective as disorder-specific CBT, which focuses on a specific cognitive behavioural model for a specific disorder (Newby et al., 2015).

CBT in Saudi Arabia has started to gain momentum among mental health practitioners and has become an attractive psychological treatment for anxiety and mood disorders. However, 
the majority of these services are based on a disorder-specific approach. Although disorder-specific CBT represents a major milestone in the progress of psychotherapy, it is not without limitations. One major limitation is that people do not always fit one set of criteria for a specific disorder. In fact, comorbidity in emotional disorders is more a common phenomenon than an exception, with $50 \%$ of clients reporting comorbid anxiety and depression (Brown and Barlow, 2002).

Moreover, the highly specialized manuals for treating different disorders (sometimes more than one manual for the same disorder) create a major barrier to treatment delivery in many countries with limited resources (Murray et al., 2014). In Saudi Arabia, there are a limited number of qualified and well-trained CBT therapists who can deliver these specialized interventions. Training opportunities have been initiated to increase this number. However, a disorder-specific approach requires training and supervision for a large number of protocols and manuals, and the limited number of qualified supervisors is a major challenge to effective training outcomes for this approach.

Therefore, T-CBT may have a practical advantage over disorder-specific CBT, especially in countries with limited resources such as Saudi Arabia. T-CBT offers the flexibility to address a wide range of disorders and comorbid conditions, therefore increasing access to psychological intervention (Clark and Taylor, 2009). T-CBT also allows training to focus on one unified protocol, thereby improving the quality and applicability of training and supervision. Additionally, T-CBT can provide a more efficient and cost-effective model of treatment in general (Bullis et al., 2015).

The first initial evidence supporting the use of the transdiagnostic approach in Saudi Arabia was a case report on four female clients suffering from a wide range of difficulties (Alatiq, 2014). This initial evidence has encouraged us to further develop the use of this approach. One of our initiatives is to close the gap between the high demand for psychotherapy and the limited number of specialized therapists through the 'Task Sharing Model' (Murray et al., 2011). This approach promises to decrease the treatment gap in countries with limited resources by building the capacity of the mental health workforce. In a study in Iraq and Thailand, this approach was used to train lay workers with little prior mental health training and experience to become counsellors providing transdiagnostic interventions. In two pilot studies, the results suggested significant improvement above the minimal threshold of depression, anxiety and post-traumatic experience (Murray et al., 2014).

In Saudi Arabia, it has been estimated that approximately 95\% of psychologists are junior, with only a bachelors or masters degree in psychology and with limited training and experience in psychotherapy (Alqahtani, 2019). This large percentage of psychologists is an important workforce in the country and should be targeted for capacity building to address the treatment gap issues. They have the basic knowledge and educational foundation for mental health issues and psychological theories in general. However, to take an active part in service delivery, they need practical training and supervision in psychological interventions that balance being appropriate for delivery by junior staff and at the same time allows the flexibility for day-to-day clinical practice.

Therefore, in this study, we aim to examine the effectiveness of T-CBT in a naturalistic setting and to train junior psychologists on the use of this protocol to treat mild to moderate cases of emotional disorders as low-intensity (LI) interventions. Data were collected as part of service development and quality assurance measures. This methodology provides opportunities to measure the effect of the intervention as it is actually conducted in front-line clinical settings. Such an approach may risk the fidelity of the study because it does not follow strict randomized controlled trial protocols, but it has the advantage of narrowing the gap between clinical research and clinical practice and it is an appropriate approach for a feasibility trial.

\section{Method}

\section{Design and procedure}

In this study, the effect of T-CBT was examined using data from naturalistic clinical settings with limited or no control over the process of matching research criteria or protocols. The data were collected as part of routine service monitoring and quality assurance efforts. The participants were 
patients seen in the psychology unit of a general hospital in Riyadh. This clinic provides general psychological services, not only CBT, such as assessments and evaluations, brief counselling, family therapy and stress management interventions. Therefore, patients typically undergo assessment during their first visit to determine the type of service they require and if the service suits their current needs. All the patients reported here were identified as suitable for CBT. Mild to moderate cases were referred to junior psychologists for LI, while complex cases were referred to a senior psychologist for a high-intensity intervention (HI). Classification of severity was mainly based on a clinical judgement of the overall evaluation of symptom types and severity. Therapists followed a protocol for the transdiagnostic approach as will be described below. Patients were informed that their data might be used for research and educational purposes if needed, and their consent was obtained. Approval and ethical consideration was obtained from the local body.

\section{Participants}

The participants involved in this study were patients who were referred to the psychology unit for psychotherapy for a wide range of difficulties. There were no strict criteria for accepting or declining a referral; the only cases declined were those with severe mental retardation or those with current active psychosis. All referred patients were seen in the Intake Clinic, where initial assessments of current difficulties were conducted. Then a decision on the distribution of the case to clinicians was made based first on suitability for CBT $v s$ other interventions, and then all CBT suitable cases were distributed to either LI or HI. Distribution was based on clinical judgement, making use of collected information about symptom levels (self-report questionnaire), the complexity of a problem (type and cluster of symptom) and/or the complexity of the case formulation. For example, symptoms of flashbacks and dissociation were considered complex cases whereas anxiety symptoms might be based on self-reported severity levels. Figure 1 shows the follow-up on referrals and the distribution of cases.

Of the 339 cases referred for psychotherapy, 198 were assigned to CBT interventions: 160 were mild to moderate cases and received LI intervention, and 38 were complex cases and therefore received $\mathrm{HI}$ intervention. A total of 141 cases were evaluated as not suitable for CBT and were

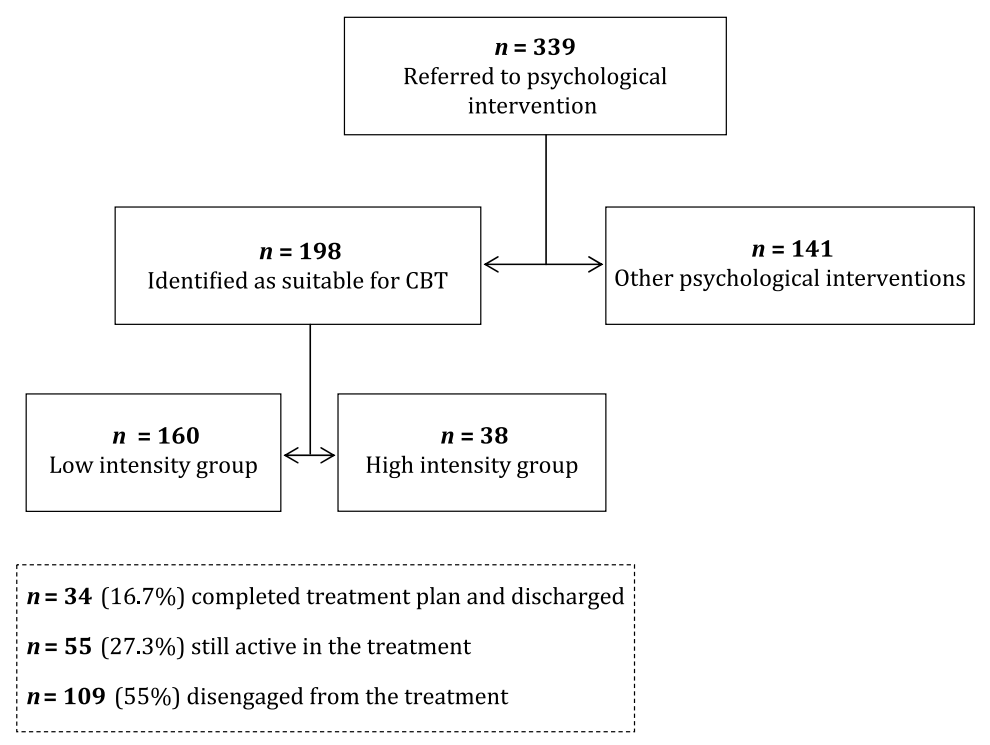

Figure 1. The follow-up of participants 
offered other forms of psychological intervention such as specific psychoeducation, counselling, supportive therapy, family therapy, or other integrative approach interventions.

The study sample consisted of all eligible patients seen between December 2015 and December 2017 despite the current status of their treatment plan (whether they completed the plan and were discharged or were still actively being followed up). Of the total number of 198 patients, $33(16.7 \%)$ completed the treatment plan and provided a post-assessment profile, and they are called Discharged patients in this report. Fifty-five patients (27.3\%) were still active in treatment by the end of the study period and are called Active patients. One hundred and nine patients (55\%) did not show up for their last appointment and/or did not make further appointments; those patients were called Disengaged patients.

\section{Measures}

To measure the baseline level of severity and to establish therapeutic outcome measures, the following self-report questionnaires were administered during the intake assessment (pre-assessment) and on discharge from service (post-assessment):

\section{Symptom Checklist-90-Revised (SCL-90-R)}

This instrument is designed to screen for a vast range of psychological complications and symptoms of psychopathology. The SCL-90-R is a multi-dimensional questionnaire consisting of nine subscales of primary symptom dimensions and it produces two total scores: a grand total (GT), ranging from 0 to 360, and the positive symptom total (PST), ranging from 0 to 90 . It also produces two global indexes that assess overall psychological distress: the global severity index (GSI) and the positive symptom distress index (PSDI), ranging from 0 to 4 (Derogatis, 1977). The Arabic version of the questionnaire was used in this study and has good psychometric properties (Albuhairi, 2005).

\section{Ferrans and Powers' Quality of Life Index (QLI)}

This is used to assess satisfaction level with the main aspects of life. The QLI consists of four domains: health and functioning, psychological/spiritual, social and economic, and family. The scale measures level of satisfaction and evaluates it against level of importance. The final overall score ranges from 0 to 30 , with higher scores suggesting higher quality of life (Ferrans and Powers, 1985).

\section{Patient Health Questionnaire (PHQ-9)}

The PHQ-9 is a widely used self-report questionnaire to determine the presence and severity of depression. The PHQ-9 consists of nine items with a total score ranging from 0 to 27 (Spitzer et al., 2006).

\section{Generalized Anxiety Disorder questionnaire (GAD-7)}

The GAD-7 is a widely used self-report questionnaire for anxiety symptoms. The questionnaire consists of seven items mainly designed for screening, diagnosing and measuring generalized anxiety disorder. The total score on the GAD-7 for the seven items ranges from 0 to 21 (Kroenke et al., 2001).

\section{Sheehan Disability Scale (SDS)}

This brief, 3-item self-administered questionnaire is designed to measure functional impairment in three main domains: work/school, social life, and family life. For each scale, 
patients rate the extent to which these domains are impaired by their symptoms from 0 to 10 (Sheehan, 1983).

\section{Clinical Global Impression (CGI)}

The CGI is a clinician rating scale for measuring severity and change. The severity measure is a 7-point scale that requires the clinician to rate the severity of the patient's symptoms at the time of assessment. The change measure is also a 7-point scale that requires the clinician to assess how much the patient's symptoms have improved or worsened relative to a baseline state. Many consider this measure objective because it is based on clinical experience and judgement (Guy, 1976).

To monitor improvement on a session-to-session basis, four assessments were also administered at the beginning of each therapy session: the PHQ, GAD, SDS and CGI-change subscales. The data used in this report for the monitoring assessments were from the first therapy session and the last therapy session.

\section{Treatment description}

The T-CBT protocol was developed by the first author based on the existing literature on this intervention, e.g. Barlow et al. (2017) and Norton (2012a). It follows the same principle of recognizing and targeting primary underlying cognitive and behavioural processes such as mindfulness awareness, emotional avoidance in Barlow et al. (2017), and exposure and response prevention in Norton (2012a). It also follows the same line in providing psychoeducation and socializing to treatment at the start of the therapy and ends with relapse prevention work. However, Norton's programme was a group design and has a list of 12 sessions that must be followed, each of which has a specific focus that is mainly around two themes: cognitive restructuring and exposure and response prevention. The programme of Barlow et al., on the other hand, is based on five core treatment modules (mindfulness emotion awareness; cognitive flexibility; emotion avoidance; emotion related physical sensations; emotion focused exposure). In their trial, therapists had to adhere to this treatment protocol and reported excellent treatment fidelity scores.

However, our programme was designed to be case formulation driven. It has broad elements of focus, rather than step-by-step manuals or specific modules to follow. The focus of therapy is selected based on the case formulation and hypothesis, which is developed and shared with the patients. This approach allows for flexibility in matching clients' needs, level of complexity and readiness to change. For example, clients who are insightful about his/her irrational ways of thinking but who cannot disengage from unhelpful avoidance patterns of behaviour might not need to work on cognitive challenges or restructuring but rather the focus is directed to exposure and behavioural work. Another example is clients who are really bothered by the confusion of 'why this is happening to me?'; they might need to spend more time sharing case formulations and understanding the underlying process that helps to develop and maintain the problem.

Our approach is rather similar in principle to the Common Element Treatment Approach (CETA) described by Murray et al. (2014), in which the counsellor can choose from a list of elements based on the client's primary problem. However, in their trial they developed a possible component of elements for different scenarios with the primary problems being either depression or trauma-related anxiety. In our trial, we did not work on developing such guidelines to allow each therapist to make decisions on an individual case-by-case basis, with the use of supervision if needed.

Developing such a programme requires accounting for cultural factors that may hinder or improve outcomes. We believe that no major modifications to the intervention are needed in 
terms of the underlying theoretical and conceptual basis but rather to the specific techniques that are applied. This is in line with the findings of Murray et al. (2014) in Iraq and Thailand; these authors reported that cultural adaptation is needed for 'peripheral aspects (e.g., terminology, analogies) and not core treatment elements' (p. 2). Therefore, and based on clinical experience, some minor modifications were added to the application of some of the techniques, which are stated in the description below and in Table 1.

The protocol consists of the following elements:

\section{(1) Assessment and case formulation}

This involves taking a full history of the patient's current and past difficulties and assessing the cognitive and behavioural processes that may have played a role in triggering or maintaining the problem. A case formulation is then developed and shared with the patient. The case formulation is based on Padesky and Mooney's (1990) generic model, which is a framework for how cognitive,

Table 1. Treatment description with cultural modification required

\begin{tabular}{|c|c|c|}
\hline Elements & Description & Cultural modifications \\
\hline $\begin{array}{l}\text { 1. Assessment and } \\
\text { case formulation }\end{array}$ & $\begin{array}{l}\text { - Taking history } \\
\text { - Assessing cognitive and behavioural } \\
\text { processes } \\
\text { - Developing and sharing case formulation }\end{array}$ & $\begin{array}{l}\text { - In developing and sharing the case } \\
\text { formulation, special attention is given } \\
\text { to the collaborative aspect of this } \\
\text { process and the fact that formulation } \\
\text { is working hypothesis is emphasized }\end{array}$ \\
\hline $\begin{array}{l}\text { 2. Psychoeducation } \\
\text { and increased } \\
\text { awareness }\end{array}$ & $\begin{array}{l}\text { - Psychoeducation about the cognitive } \\
\text { behavioural model } \\
\text { - Reviewing the longitudinal aspects of the } \\
\text { development in light of the CBT model } \\
\text { - Increasing awareness of the underlying } \\
\text { processes through simple and informal } \\
\text { mindfulness techniques }\end{array}$ & $\begin{array}{l}\text { - An important step in socializing } \\
\text { patients to the interventions, } \\
\text { particularly if a client has no prior } \\
\text { knowledge or experience with } \\
\text { psychotherapy and CBT }\end{array}$ \\
\hline $\begin{array}{l}\text { 3. Cognitive } \\
\text { approach }\end{array}$ & $\begin{array}{l}\text { - Identifying the content of thoughts and } \\
\text { patterns of assumptions and } \\
\text { interpretations } \\
\text { - Cognitive restructuring work in session. } \\
\text { - Encouraging monitoring and challenging } \\
\text { thought between sessions } \\
\text { - Identifying cognitive processes that } \\
\text { maintain the symptoms (e.g. thought } \\
\text { suppression, rumination, and self-focused } \\
\text { attention) } \\
\text { - Encouraging observing and monitoring } \\
\text { the effect of such processes } \\
\text { - Encouraging developing more adaptive } \\
\text { cognitive responses through mindfulness } \\
\text { practice }\end{array}$ & $\begin{array}{l}\text { - Use more culturally relevant } \\
\text { discussions and culture-specific } \\
\text { metaphors } \\
\text { - Link mindfulness techniques to spiritual } \\
\text { and religious practices }\end{array}$ \\
\hline $\begin{array}{l}\text { 4. Behavioural } \\
\text { approach }\end{array}$ & $\begin{array}{l}\text { - Identifying safety behaviours } \\
\text { - Discussing the role played by escape and } \\
\text { avoidance in maintaining symptoms and } \\
\text { their underlying beliefs } \\
\text { - Encouraging behavioural experiments to } \\
\text { examine and test alternative thoughts } \\
\text { and behavioural responses }\end{array}$ & $\begin{array}{l}\text { Emphasize the active participation of } \\
\text { the client in designing any behavioural } \\
\text { experiments or challenges. This is to } \\
\text { maintain the collaborative role of the } \\
\text { client in therapy (not being a passive } \\
\text { recipient of homework assignments) }\end{array}$ \\
\hline $\begin{array}{l}\text { 5. Termination and } \\
\text { relapse } \\
\text { prevention }\end{array}$ & $\begin{array}{l}\text { - Work on termination issues } \\
\text { - Identifying successful aspects of } \\
\text { treatment } \\
\text { - Developing a relapse prevention plan }\end{array}$ & $\begin{array}{l}\text { Discuss the time limited work of } \\
\text { therapy early on to prepare clients for } \\
\text { discharge and to acknowledge their } \\
\text { active role in therapy and beyond }\end{array}$ \\
\hline
\end{tabular}


behavioural and environmental factors interact with each other to maintain current difficulties. One addition was made to this model, which emphasizes cognitive content $v s$ cognitive processes. This is to incorporate the common cognitive processes suggested by Harvey et al. (2004) such as rumination, thought suppression and attentional biases.

The most important cultural consideration in this part is the delivery process of case formulation. Special attention is given to the collaborative aspect of this step so that the formulation is shared as a working hypothesis rather than the ultimate answer to the cause of the problem. This approach is highly important, as many clients may not be familiar with the collaborative role and the systemic process of hypotheses testing in CBT. This is also a particularly important aspect of training for CBT vs other forms of therapy. Trainees who are familiar with other forms of therapy such as psychodynamic approaches, might tend to hold any impression about case formulation or interpretation until they believe they have reached the right moment in the therapy process. This is a significant difference of the CBT approach in which impressions about case formulation are discussed early on in therapy as hypotheses to be tested. Such differences in methodology need to be highlighted and explicitly discussed, especially if trainees or clients have had any previous experience with other forms of therapy.

(2) Psychoeducation and increased awareness

Comprehensive psychoeducation about the nature of the patient's difficulties and the underlying process is a major part of the treatment protocol. This includes providing educational materials about the cognitive behavioural model of the problem and reviewing the longitudinal aspect of the development of the problem in light of this model. One important goal of the psychoeducation is to increase awareness of this underlying process, which is accomplished by asking the patients to start observing this kind of link and to continue monitoring and recording these observations. Simple and informal mindfulness techniques such as 'noticing' could be used at this stage to increase such awareness.

This part of the protocol is considered an important step in socializing patients to the interventions. It is particularly important in a culture in which knowledge and understanding of CBT might not be as common as in a Western culture, where CBT was originally founded.

\section{(3) Cognitive approach}

This step focuses on two aspects. The first involves identifying the content of thoughts by discussing the patterns of assumptions and interpretations of significant events. The participants are encouraged to identify their maladaptive beliefs and misinterpretations by monitoring their thoughts. Then, cognitive restructuring and working towards more adaptive alternative interpretations is introduced and worked through in session using Socratic dialogues. There is no cultural specification for this step except the use of more relevant discussion and culture-specific metaphors.

Second, the cognitive processes that maintain the symptoms are identified by discussing the role of thought suppression, rumination and self-focused attention. The aim is to encourage patients to observe and monitor the effect of such processes on their current mood and symptoms to develop more adaptive cognitive responses. Such responses are achieved by using mindfulness techniques to view thoughts as mental events rather than reality; and to facilitate acceptance and letting go as opposed to rumination and suppression. The mindfulness technique has been found to be helpful and relevant when it is linked to spiritual and religious practices; for example, clients pray five times a day and find this to be a useful opportunity to incorporate mindfulness, not only for the sake of mind training but also for its potential value to improve the spiritual experience of the prayer. 
(4) Behavioural approach

This step focuses on identifying safety behaviour and the role played by escape and avoidance in maintaining symptoms and their underlying thoughts and beliefs. In addition, behavioural experiments are also used to examine and test alternative thoughts and behaviours. One cultural consideration is the need to emphasize the active participation of the clients in this process. Patients need to be encouraged to participate in designing the behavioural experiments in order to continue assuming a collaborative role rather than acting as a passive recipient of homework assignments.

\section{(5) Termination and relapse prevention}

This is the final step of the treatment process and focuses on termination issues, the identification of the successful aspects of treatment, and the development of a relapse prevention plan. Relapse prevention involves the identification of triggers and other factors that might suggest relapse and the development of an effective action plan. One cultural consideration here is to introduce the time limited frame of the therapy and the concept of relapse prevention at early stages in therapy. This is to prepare the client that this work will come to an end and that they need to take an active part in the process of therapy as well as maintaining improvement.

As described earlier, the mild-to-moderate cases received the protocol as an LI intervention, while complex cases received the same protocol as a $\mathrm{HI}$ intervention. The main difference between the two interventions was the level of depth and complexity in following the protocol. HI was mainly used for more complex cases and therefore required a more complex case formulation and advanced cognitive and behavioural interventions. Other than that, the goal and process are similar.

\section{Therapists}

The therapists involved in this study included one senior psychologist and six junior psychologists with a first degree in psychology and 2-5 years of experience working in the psychology unit. One of the junior psychologists had a postgraduate diploma in CBT from a local programme, and one had a master's degree in clinical psychology from a Western university with a focus on counselling skills. Some had attended local workshops in CBT.

All the therapists received one week of intensive training on the use of this protocol and continued to receive individual and group supervision on a weekly basis by the senior psychologist (the first author). Competencies were measured using the Cognitive Therapy Rating Scale (CTRS) (Young and Beck, 1980) to assess the therapists' skills in sessions using audiotape recordings. Although the plan involved performing weekly audiotape recordings for each therapist, the patients' refusal to allow recording prevented this. Alternatively, at least three audiotapes for each therapist were collected, confirming that he/she was following the treatment protocol and had adequate CBT skills.

\section{Results}

\section{Sample description}

Table 2 shows all the demographic data for the sample as well as information about diagnoses and the services received. As shown in Table 2, there were no significant differences between the patients in the LI group and the patients in the HI group for any of the demographic variables such as age, gender, marital status and educational level.

However, regarding primary diagnosis, there was a significant difference between the groups $(x=36.1$ (9), $p=.000)$. Compared with the HI group, the LI group had more patients with 
Table 2. Demographics, diagnoses and service related information

\begin{tabular}{|c|c|c|c|c|}
\hline Demographics & $\begin{array}{c}\text { Overall } \\
\text { sample } \\
(n=198)\end{array}$ & $\begin{array}{c}\text { High } \\
\text { intensity } \\
(n=38)\end{array}$ & $\begin{array}{c}\text { Low } \\
\text { intensity } \\
(n=160)\end{array}$ & Difference \\
\hline Age (mean, $S D$ ) & $32.2(10.1)$ & $34.6(10.4)$ & $31.6(10.1)$ & $t=-1.58(192), p=.115$ \\
\hline Female & $134(67.7)$ & $26(68.4)$ & $108(67.5)$ & $x=.012(1), p=.913$ \\
\hline \multicolumn{5}{|l|}{ Marital status } \\
\hline$\circ$ Single & $70(35.4)$ & $16(42.1)$ & $54(33.7)$ & \multirow[t]{4}{*}{$x=2.85(3), p=.416$} \\
\hline - Married & $103(52.0)$ & $20(52.6)$ & $83(51.9)$ & \\
\hline$\circ$ Divorced & $4(2.0)$ & $0(0)$ & $4(2.5)$ & \\
\hline$\circ$ Widow & $14(7.1)$ & $1(2.6)$ & $13(8.1)$ & \\
\hline $\begin{array}{l}\text { Family members } \\
\text { (mean, SD) }\end{array}$ & $6.1(2.6)$ & $6.2(2.6)$ & $6.1(2.6)$ & $t=-.186(147), p=.853$ \\
\hline Children (mean, SD) & $2.1(2.3)$ & $3.2(3.4)$ & $2.0(2.1)$ & $t=.178(134), p=.076$ \\
\hline \multicolumn{5}{|l|}{ Educational } \\
\hline - Illiterate & $4(2.0)$ & $0(0)$ & $4(2.5)$ & \multirow[t]{8}{*}{$x=9.55(7), p=.215$} \\
\hline - Read and write & $1(0.5)$ & $1(2.6)$ & $0(0)$ & \\
\hline$\circ$ Primary & $2(1.0)$ & $0(0)$ & $2(1.2)$ & \\
\hline - Secondary & $18(9.1)$ & $1(2.6)$ & $17(10.6)$ & \\
\hline - High school & $53(26.8)$ & $9(23.7)$ & $44(27.5)$ & \\
\hline - Diploma & $18(9.1)$ & $2(5.3)$ & $16(10.0)$ & \\
\hline - University & $53(26.8)$ & $8(21.0)$ & $45(28.1)$ & \\
\hline - Higher education & $4(2.0)$ & $0(0)$ & $4(2.5)$ & \\
\hline \multicolumn{5}{|l|}{ Employment } \\
\hline - Not working & $106(53.5)$ & $11(28.9)$ & $95(59.4)$ & \multirow[t]{3}{*}{$x=1.44(2), p=.695$} \\
\hline - Government sector & $53(26.8)$ & $8(21.0)$ & $45(28.1)$ & \\
\hline$\circ$ Private sector & $4(2.0)$ & $0(0)$ & $4(2.5)$ & \\
\hline \multicolumn{5}{|l|}{ Diagnosis and onset } \\
\hline \multicolumn{5}{|l|}{ Primary diagnosis: } \\
\hline - Depression & $61(30.8)$ & $9(23.7)$ & $52(32.5)$ & \multirow[t]{9}{*}{$x=36.1(9), p=.000$} \\
\hline$\circ$ Anxiety & $58(29.3)$ & $6(15.8)$ & $52(32.5)$ & \\
\hline$\circ \mathrm{OCD}$ & $24(12.1)$ & $5(13.1)$ & 19 (11.9) & \\
\hline - Panic & $21(10.6)$ & $4(10.5)$ & $17(10.6)$ & \\
\hline - Social & $19(9.6)$ & $4(10.5)$ & $15(9.4)$ & \\
\hline$\circ \mathrm{PTSD}$ & $5(2.5)$ & $3(7.9)$ & $2(1.2)$ & \\
\hline - Conversion & $4(2.0)$ & $4(10.5)$ & $0(0)$ & \\
\hline$\circ$ Phobia & $2(1.0)$ & $1(2.6)$ & $1(0.6)$ & \\
\hline$\circ$ Complicated grief & $2(1.0)$ & $0(0)$ & $2(1.2)$ & \\
\hline \multicolumn{5}{|l|}{ Number of diagnoses } \\
\hline$\circ$ Only one primary & $127(64.1)$ & $33(86.8)$ & $94(58.7)$ & \multirow[t]{2}{*}{$x=10.5(1), p=.001$} \\
\hline - Two or more & 71 (35.9) & $5(13.1)$ & $66(41.2)$ & \\
\hline \multicolumn{5}{|l|}{ Onset } \\
\hline$\circ$ Less than 1 year & $45(22.7)$ & $8(21.0)$ & $37(23.1)$ & \multirow[t]{4}{*}{$x=1.83(3), p=.608$} \\
\hline$\circ$ 2-5 years & $60(30.3)$ & $10(26.3)$ & $50(31.2)$ & \\
\hline$\circ 6-10$ years & $22(11.1)$ & $3(7.9)$ & 19 (11.9) & \\
\hline - More than 10 years & $37(18.7)$ & $3(7.9)$ & $34(21.2)$ & \\
\hline \multicolumn{5}{|c|}{$\begin{array}{l}\text { Service related information } \\
\text { Psychiatry }\end{array}$} \\
\hline$\circ$ Yes & $120(60.6)$ & $31(81.6)$ & $89(55.6)$ & \multirow[t]{2}{*}{$x=9.41(2), p=.009$} \\
\hline$\circ \mathrm{No}$ & $39(19.7)$ & $2(5.3)$ & $37(23.1)$ & \\
\hline \multicolumn{5}{|l|}{ Status } \\
\hline$\circ \mathrm{AC}$ & $55(27.3)$ & $10(23.7)$ & $45(28.1)$ & \multirow[t]{3}{*}{$x=3.54(2), p=.315$} \\
\hline$\circ N S$ & $109(55.1)$ & $19(50.0)$ & $90(56.2)$ & \\
\hline$\circ D C$ & $33(16.7)$ & $10(26.3)$ & $23(14.4)$ & \\
\hline $\begin{array}{l}\text { Number of sessions } \\
\text { (mean, SD) }\end{array}$ & $5.3(4.7)$ & $6.3(4.3)$ & $5.0(4.9)$ & $t=1.52(195), p=.130$ \\
\hline $\begin{array}{l}\text { Number of missed } \\
\text { appointments }\end{array}$ & $1.8(1.8)$ & $0.87(1.6)$ & $2.02(1.8)$ & $t=3.65(198), p=.000$ \\
\hline $\begin{array}{l}\text { Months in service } \\
\text { (mean, SD) }\end{array}$ & $3.5(4.5)$ & $4.4(5.5)$ & $3.3(4.1)$ & $t=1.31(196), p=.190$ \\
\hline
\end{tabular}

Results are presented as number (\%) unless stated. 
depression (32 vs 23\%) and anxiety (32 vs 16\%) as the primary diagnosis, and fewer patients with PTSD (1 vs 8\%) and conversion disorders (0 vs 10\%).

For services received, the HI group had more patients receiving psychiatric care $(81 \%)$ compared with the LI group (55\%) $(x=9.41(2), p=.009)$. Moreover, the groups differed in the number of missed appointments $(t=3.65(198), p=.000)$, with the patients in the LI group missing more appointments $(2.02, S D$ 1.8) than the HI group $(0.87, S D 1.6)$.

\section{Baseline assessments}

Table 3 shows all the baseline assessment measures for the overall sample as well as the groups. Although the HI group had elevated symptoms based on higher scores on all SCL-90-R scales and lower scores on the QLI measures, these differences were not significantly different. However, there was a significant difference between the groups on the PHQ $(t=2.70$ (135), $p=.008)$ and the GAD ( $t=1.97$ (135), $p=.050)$ : the HI group had higher scores, indicating more severe depression and anxiety symptoms. Additionally, there was a significant difference in the clinicians' ratings of severity using the CGI measure $(x=16.2(6) ; p=.012)$, with $31 \%$ of the HI group evaluated in the severe category, while $31 \%$ of the patients in the LI group were evaluated in the moderate category.

\section{General symptoms and quality of life}

Table 4 shows the effect size (Cohen's $d$ ) difference from the pre-assessment to the post-assessment results for patients who completed the treatment plan and provided a post-assessment profile on the SCL-90-R and QLI measures. The results indicated a large effect size (range: 0.55 to 1.84) for all subscales and total measures from the two questionnaires (the SCL-90 and QLI). This result held for the overall sample $(n=33)$ as well as for the LI group $(n=23)$.

Table 3. Baseline assessment

\begin{tabular}{|c|c|c|c|c|}
\hline Scale & $\begin{array}{l}\text { Overall } \\
\text { sample } \\
(n=198)\end{array}$ & $\begin{array}{c}\text { High } \\
\text { intensity } \\
(n=38)\end{array}$ & $\begin{array}{c}\text { Low } \\
\text { intensity } \\
(n=160)\end{array}$ & Difference \\
\hline SCL-Grand Total Score & $151.5(65.2)$ & $171.8(89.7)$ & $150.1(63.5)$ & $t=.909(124), p=.365$ \\
\hline SCL-Positive Symptoms Total & $63.5(20.3)$ & $74.4(14.4)$ & $62.8(20.5)$ & $t=1.57(125), p=.120$ \\
\hline SCL-Global Severity Index & $1.79(0.70)$ & $2.1(0.75)$ & $1.8(0.69)$ & $t=1.43(121), p=.155$ \\
\hline SCL-Symptoms Distress Index & $2.28(0.49)$ & $2.54(0.68)$ & $2.3(0.48)$ & $t=1.52(122), p=.130$ \\
\hline QLI-Total Score & $16.9(6.1)$ & $13.1(7.3)$ & $17.1(6.0)$ & $t=1.55(118), p=.124$ \\
\hline QLI-Health \& Function & $16.0(6.4)$ & $13.3(8.3)$ & $16.2(6.3)$ & $t=1.152(121), p=.252$ \\
\hline QLI-Social \& Economic & $16.2(6.8)$ & $15.9(6.5)$ & $16.2(6.8)$ & $t=.126(121), p=.900$ \\
\hline QLI-Psychological \& Spiritual & $16.9(7.6)$ & $11.7(8.5)$ & $17.2(7.5)$ & $t=1.88(119), p=.062$ \\
\hline QLI-Family & $19.8(7.2)$ & $16.7(7.1)$ & $18.1(7.3)$ & $t=1.16(121), p=.249$ \\
\hline PHQ & $12.9(5.8)$ & $18.6(4.0)$ & $12.6(5.7)$ & $t=2.70(135), p=.008$ \\
\hline GAD & $10.3(5.6)$ & $14.3(6.3)$ & $10.0(5.5)$ & $t=1.97(135), p=.050$ \\
\hline SDS & $16.6(7.5)$ & $18.6(9.4)$ & $16.5(7.4)$ & $t=.239(211), p=.881$ \\
\hline \multicolumn{5}{|l|}{ CGI-Severity $n(\%)$} \\
\hline$\circ$ Normal & $1(0.5)$ & $0(0)$ & $1(0.6)$ & $x=16.2(6), p=.012$ \\
\hline - Borderline & $13(6.6)$ & $0(0)$ & $13(8.1)$ & \\
\hline$\circ$ Mild & $48(24.2)$ & $2(5.3)$ & $46(28.7)$ & \\
\hline - Moderate & $57(28.8)$ & $7(18.4)$ & $50(31.2)$ & \\
\hline$\circ$ Markedly & $12(6.1)$ & 12 (31.6) & - & \\
\hline$\circ$ Severely & $5(2.5)$ & $5(13.1)$ & - & \\
\hline$\circ$ Extremely severe & - & - & - & \\
\hline
\end{tabular}

Results are presented as means $(S D)$ unless stated. 
Table 4. General symptoms and quality of life

\begin{tabular}{|c|c|c|c|c|c|c|}
\hline \multirow[b]{2}{*}{ Scale } & \multicolumn{3}{|c|}{$\begin{array}{l}\text { Overall sample completed } \\
\text { the programme }(n=33)\end{array}$} & \multicolumn{3}{|c|}{$\begin{array}{l}\text { Low intensity group completed } \\
\text { the programme }(n=23)\end{array}$} \\
\hline & Pre & Post & Effect size $(95 \% \mathrm{Cl})$ & Pre & Post & Effect size $(95 \% \mathrm{Cl})$ \\
\hline SCL-GT & $172.1(64.4)$ & $76.0(59.4)$ & 1.55 (0.98 to 2.08 ) & $170.4(65.8)$ & $75.4(61.1)$ & $1.50(0.82$ to 2.12$)$ \\
\hline SCL-PST & $71.7(19.1)$ & $47.5(29.5)$ & 0.97 (0.45 to 1.47$)$ & $71.1(19.1)$ & $46.4(29.9)$ & $0.98(0.36$ to 1.58$)$ \\
\hline SCL-GSI & $2.1(0.7)$ & $0.9(0.6)$ & 1.84 (1.25 to 2.39$)$ & $1.96(0.7)$ & $0.9(0.6)$ & $1.63(0.93$ to 2.26$)$ \\
\hline SCL-PSDI & $2.3(0.42)$ & $1.4(0.60)$ & 1.74 (1.15 to 2.28$)$ & $2.3(0.43)$ & $1.4(0.60)$ & $1.72(1.30$ to 0.11$)$ \\
\hline QLI-T & $17.3(6.1)$ & $22.2(7.2)$ & 0.73 (1.22 to 0.23$)$ & $17.3(6.1)$ & $22.1(7.3)$ & 0.71 (1.16 to 0.01$)$ \\
\hline QLI-H & $16.8(6.1)$ & $20.8(7.5)$ & 0.59 (1.07 to 0.09$)$ & $16.8(6.1)$ & $20.8(7.5)$ & 0.59 (1.32 to 0.12$)$ \\
\hline QLI-S & $15.1(7.2)$ & $20.6(7.8)$ & $0.73(1.22$ to 0.23$)$ & $15.1(7.2)$ & $20.6(7.8)$ & $0.73(1.52$ to 0.30$)$ \\
\hline QLI-P & $16.0(7.4)$ & $22.9(7.5)$ & 0.93 (1.42 to 0.41$)$ & $16.0(7.4)$ & $22.9(7.5)$ & $0.93(1.52$ to 0.30$)$ \\
\hline QLI-F & $18.5(8.3)$ & $22.9(7.9)$ & 0.54 (1.03 to 0.05 ) & $18.5(8.3)$ & $22.9(7.8)$ & 0.55 (1.13 to 0.05$)$ \\
\hline
\end{tabular}

SCL-GT, Total Score; SCL-PST, Positive Symptom Total; GSI, Global Severity Index; SCL-PSDI, Positive Symptom Distress Index; QLI-T, Total; QLI-H, Health and Functioning; QLI-S, Social and Economic; QLI-P, Psychological/Spiritual; QLI-F, Family.

Table 5. Depression, anxiety and function

\begin{tabular}{|c|c|c|c|c|c|c|c|}
\hline \multicolumn{8}{|c|}{ Overall sample ( $n=198)$} \\
\hline Scale & Pre & $\begin{array}{c}\text { First } \\
\text { session }\end{array}$ & $\begin{array}{c}\text { Last } \\
\text { session }\end{array}$ & Post & $\begin{array}{l}\text { Pre- to first } \\
\text { effect size } \\
(n=198)\end{array}$ & $\begin{array}{l}\text { Pre- to last } \\
\text { effect size } \\
(n=198)\end{array}$ & $\begin{array}{l}\text { Pre-post } \\
\text { effect size } \\
(n=33)\end{array}$ \\
\hline PHQ & $12.9(5.8)$ & $12.0(6.0)$ & $10.1(5.7)$ & $7.7(5.7)$ & $0.15(0.05$ to 0.35$)$ & $0.49(0.29$ to 0.69$)$ & $0.90(0.52$ to 1.27$)$ \\
\hline GAD & $10.3(5.6)$ & $10.4(5.6)$ & $8.7(5.6)$ & $5.4(5.2)$ & $0.02(0.21$ to 0.18$)$ & $0.29(0.09$ to 0.48$)$ & $0.88(0.50$ to 1.26$)$ \\
\hline SDS & $16.6(7.5)$ & $15.4(7.3)$ & $12.8(7.2)$ & $8.6(6.6)$ & 0.16 (0.04 to 0.36$)$ & 0.52 (0.32 to 0.72$)$ & 1.08 (0.70 to 1.46$)$ \\
\hline \multicolumn{8}{|c|}{ Low intensity group $(n=160)$} \\
\hline Scale & Pre & $\begin{array}{c}\text { First } \\
\text { session }\end{array}$ & $\begin{array}{c}\text { Last } \\
\text { session }\end{array}$ & Post & $\begin{array}{l}\text { Pre- to first } \\
\text { effect size } \\
(n=160)\end{array}$ & $\begin{array}{l}\text { Pre- to last } \\
\text { effect size } \\
(n=160)\end{array}$ & $\begin{array}{l}\text { Pre-post } \\
\text { effect size } \\
(n=23)\end{array}$ \\
\hline PHQ & $12.6(5.7)$ & $11.9(6.1)$ & $10.3(5.6)$ & $7.7(5.7)$ & $0.12(0.32$ to 0.56$)$ & $0.40(0.04$ to 0.84$)$ & $0.86(0.41$ to 1.30$)$ \\
\hline GAD & $10.0(5.1)$ & $10.2(5.4)$ & $8.7(5.5)$ & $5.4(5.2)$ & $0.04(0.48$ to 0.40$)$ & 0.25 (0.19 to 0.69$)$ & $0.90(0.45$ to 1.34$)$ \\
\hline SDS & $16.5(7.4)$ & $15.5(7.4)$ & $12.9(7.3)$ & $8.7(6.6)$ & $0.14(0.30$ to 0.57$)$ & $0.49(0.05$ to 0.93$)$ & $1.07(0.61$ to 1.51$)$ \\
\hline
\end{tabular}

\section{Depression, anxiety and function}

Table 5 shows the effect size (Cohen's $d$ ) difference for the PHQ, GAD and SDS as measured at four points in time: pre-assessment, first therapy session, last therapy session and post-assessment at discharge. This effect size is for the overall sample and the LI group.

The effect size between the pre-assessment to the first therapy session was small for the overall sample ( $n=198$ : PHQ 0.15 , GAD 0.02 and SDS 0.16$)$ and for the LI subgroup $(n=160$ : PHQ 0.12, GAD 0.04 and SDS 0.14).

From the pre-assessment to the last therapy session, the effect size was medium to large for the overall sample ( $n=198$ : PHQ 0.49, GAD 0.29 and SDS 0.52 ), and small to medium for the LI subgroup ( $n=198$ : PHQ 0.40, GAD 0.25 and SDS 0.49).

When comparing the pre- and post-assessment sessions for patients who completed the programme, the results showed a large effect size in all measures for the overall sample $(n=33$ : PHQ 0.90, GAD 0.88 and SDS 1.08) and the LI group ( $n=23$ : PHQ 0.86, GAD 0.90 and SDS 1.07).

\section{Clinical impression of change}

Table 6 shows the results for clinical impression of change using the CGI for the overall sample and the LI intervention at two time points: the last therapy session and post-assessment 
Table 6. Clinical impression of change

\begin{tabular}{lccccc}
\hline & \multicolumn{2}{c}{ Overall sample } & & \multicolumn{2}{c}{ Low intensity group } \\
\cline { 2 - 3 } CGI-Change & $\begin{array}{c}\text { Last session } \\
(n=198)\end{array}$ & $\begin{array}{c}\text { Post } \\
(n=33)\end{array}$ & & $\begin{array}{c}\text { Last session } \\
(n=160)\end{array}$ & $\begin{array}{c}\text { Post } \\
(n=23)\end{array}$ \\
\hline Improved & $133(67.2)$ & $22(66.7)$ & - & $107(67.4)$ & $21(90.8)$ \\
$\circ$ No change & $31(15.7)$ & - & $24(15.0)$ & - \\
$\circ$ Worse & $2(1.0)$ & $2(1.3)$ & - \\
\hline
\end{tabular}

Results are reported as number (\%).

at discharge. As indicated, $67 \%$ of the patients in the overall sample were evaluated as showing improvement in the last therapy session $(n=198)$ and at discharge $(n=33)$. The results for the LI group were similar in terms of the last therapy session $(n=160)$. However, this number increased to $90 \%$ for patients who completed the plan and were discharged from treatment $(n=23)$.

Only $1 \%$ of the patients were evaluated as becoming worse at the last therapy session for the overall sample $(n=198)$ and the LI group $(n=160)$, and only $15 \%$ showed no change. It is important to remember these numbers come from the entire sample, which includes clients who were still active in treatment as well as those who disengaged.

\section{Results by status (discharged, active and disengaged)}

As stated in the study design, the samples were collected within a timeframe despite their current status in therapy (discharged, $n=33$; active, $n=55$; or disengaged, $n=109$ ). There were no significant differences among the groups for any of the demographic variables, diagnoses and baseline assessment. However, it is expected that these groups are different in terms of service-related information, for example: number of sessions $(F=15.66(2), p=.000)$, number of missed appointments $(F=15.33(2), p=.000)$ and number of months receiving services $(F=7.58$ (196), $p=.001$ ). The discharged patients had more sessions (discharged: 9.1; disengaged: 4.1) and stayed in treatment longer (discharged: 6.2; disengaged: 3.1). The mean number of missed appointments was higher in the disengaged group than in the other groups (discharged: 1.4; disengaged: 2.4 ).

Table 7 shows the effect size between the pre- and the first assessment, and between the pre- and the last session, for patients according to their status group. Between the pre-assessment and the first assessment, all effect sizes were in the small range. Comparisons between the pre-assessment and last assessment showed the following pattern: the discharged group had

Table 7 . Results by status (discharged, disengaged and active)

\begin{tabular}{|c|c|c|c|c|c|}
\hline Scale & Pre & First session & Last session & Pre- to first effect size & Pre- to last effect size \\
\hline \multicolumn{6}{|c|}{ Discharged $(n=33)$} \\
\hline PHQ & $11.7(5.7)$ & $11.5(5.9)$ & $6.3(4.1)$ & 0.03 (0.45 to 0.52$)$ & 1.09 (0.56 to 1.59$)$ \\
\hline GAD & $9.0(5.7)$ & $9.0(5.7)$ & $6.1(5.9)$ & 0.00 (0.48 to 0.48$)$ & 0.50 (0.00 to 0.98$)$ \\
\hline SDS & $14.1(4.6)$ & $14.1(6.1)$ & $9.5(6.8)$ & $0.00(0.48$ to 0.48$)$ & 0.79 (0.28 to 1.28$)$ \\
\hline \multicolumn{6}{|c|}{ Disengaged $(n=109)$} \\
\hline $\mathrm{PHQ}$ & $13.6(5.7)$ & $12.0(6.2)$ & $12.3(5.6)$ & 0.27 (0.00 to 0.53$)$ & $0.23(0.04$ to 0.50$)$ \\
\hline GAD & $11.3(5.8)$ & $10.5(5.8)$ & $9.7(5.3)$ & 0.14 (0.13 to 0.40$)$ & $0.29(0.02$ to 0.55$)$ \\
\hline SDS & $17.1(7.7)$ & $15.2(7.3)$ & $13.4(7.1)$ & 0.25 (0.01 to 0.52$)$ & 0.50 (0.23 to 0.77$)$ \\
\hline \multicolumn{6}{|c|}{ Active $(n=55)$} \\
\hline PHQ & $12.7(5.1)$ & $12.1(5.5)$ & $10.0(5.7)$ & $0.11(0.27$ to 0.49$)$ & 0.50 (0.11 to 0.88$)$ \\
\hline GAD & $10.0(5.4)$ & $10.0(5.4)$ & $9.4(5.4)$ & $0.00(0.38$ to 0.38$)$ & $0.11(0.27$ to 0.49$)$ \\
\hline SDS & $16.6(6.5)$ & $15.8(7.1)$ & $13.5(7.3)$ & $0.12(0.26$ to 0.49$)$ & 0.45 (0.06 to 0.83$)$ \\
\hline
\end{tabular}



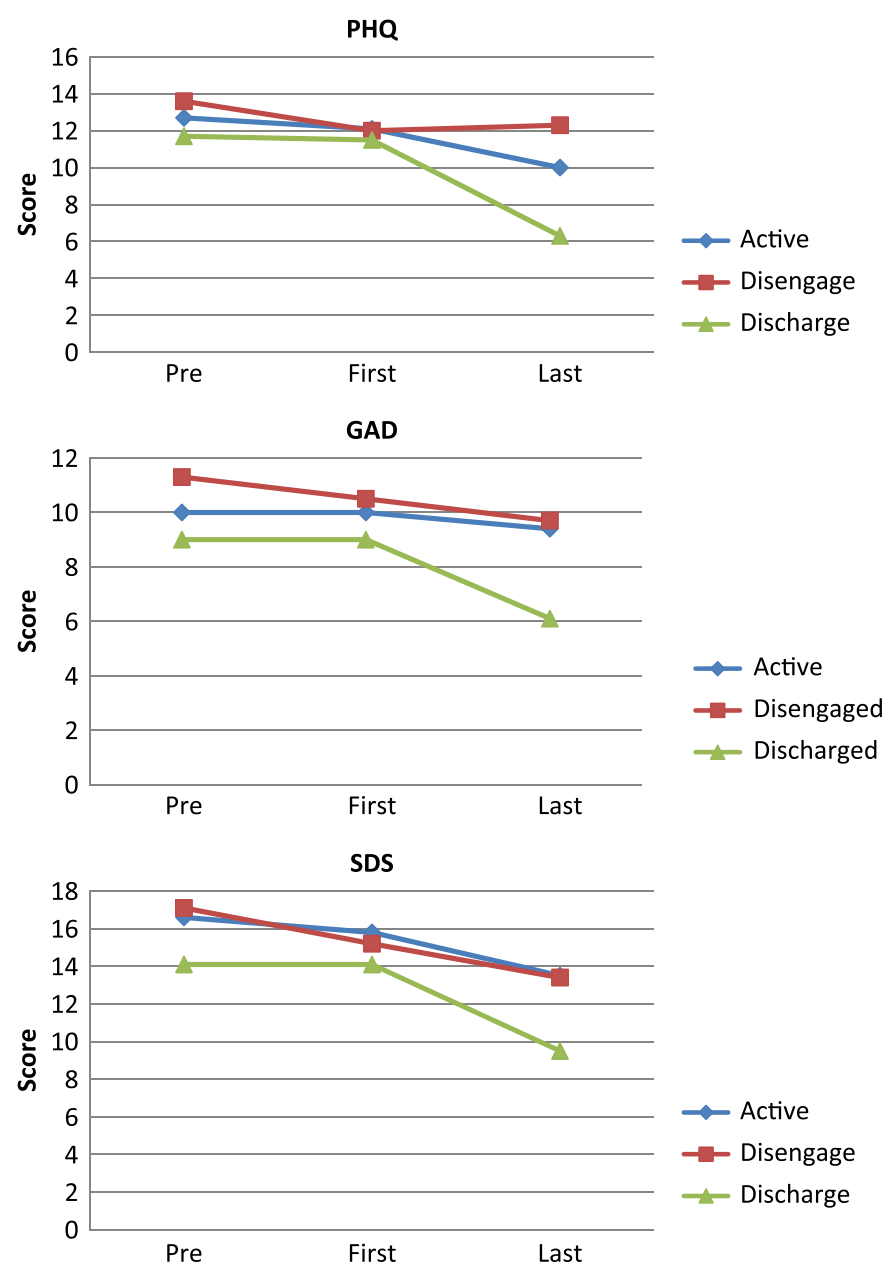

Figure 2. Profile plots for status group $\times$ time on PHQ, GAD and SDS

the largest effect size in all measures, and the disengaged group had a small effect size for depression, a medium effect size for anxiety and a large effect size for dysfunction.

Profile plots of status by time for all three measures in Fig. 2 show that all the groups had a reduction in symptoms for all measures between the pre-, first and last sessions. However, the largest change was observed for patients who completed the programme and were discharged properly from treatment.

\section{Discussion}

The application of T-CBT in Saudi Arabia warrants examination of its feasibility and effectiveness. In this study, we used an open trial approach to examine the effect of T-CBT for adults with emotional disorders. The data were collected in a naturalistic setting as part of service development and quality assurance efforts; therefore, there was no strict protocol for acceptance criteria or allocation processes. This methodology provides opportunities to measure the effect of this intervention, as it is actually conducted in front-line clinical settings, which limits the gap between research and practice. To the best of our knowledge, this is the first published trial on the use of T-CBT (and CBT at large) in Saudi Arabia. 
The overall results of this study provide initial evidence on the applicability and feasibility of applying T-CBT as adapted to Saudi patients. The analyses showed a large to medium effect size for all the treatment measures when comparing different points in time and level of intervention. The robust findings come from patients who completed the treatment as planned and provided proper post-assessment profiles. This large effect was reported for all the assessment measures (general symptoms, quality of life, depression and anxiety symptoms, level of dysfunction and clinical rating of change).

The assessments were also collected from each therapy session for all patients, regardless of whether they completed the plan and were discharged or just disengaged from the treatment. This provides significant information about the patient's progress in therapy and sheds light on the status of the clients who chose to disengage from the treatment. The comparison between the pre-assessment and the last therapy session showed a large effect size for depression, anxiety and level of dysfunction in clients who completed the programme and were discharged. For the disengaged group, however, the result was a small effect size for depression, a medium effect size for anxiety and a large effect size for the reported level of daily dysfunction. This finding suggests that receiving minimal sessions of T-CBT (an average of four sessions for the disengaged group), is helpful for reducing the symptoms of anxiety and improving the level of function even if the patient does not complete the treatment plan.

This is an important finding especially because patients who disengage from therapy are always viewed as non-compliant or resistant to treatment and might question the validity of the intervention. However, in recent years, more attention has been given to the patient's perspective in the treatment plan, as well as their preferences and values, including how some clients might require a different number of sessions than other clients; therefore, these patients may choose to disengage from therapy if they feel they have received enough (Carrey, 2016). In this view, it is hard to regard disengagement as sign of resistance or non-compliance. It is rather the preference of these patients even if it is not in line with the therapist's treatment plan.

The comparisons between the discharged and disengaged groups also showed non-significant lower baseline symptoms among the discharged group and higher baseline symptoms among the disengaged group. It is therefore possible that clients who do not complete the programme and choose to disengage from therapy have more severe conditions to start with; while this was not a significant difference in our study, it warrants further examination. It is worth asking, then, is it possible that higher levels of symptoms make clients disengage from therapy earlier? It could be that for such clients, the therapist sets higher goals that require longer planning than what the client needs. Our study showed a large effect size for improving daily function but medium to small effect sizes for depression and anxiety. We could hypothesize, then, that the client's goal was to improve function while the therapist's goal was focused on reducing symptoms; therefore, once the client meets their needs (which is to improve function), they disengage from therapy. This remains a hypothesis to be tested in future studies. It would be interesting to measure patients' accounts, feedback and points of view regarding treatment content, length and satisfaction.

Moreover, the profile of the disengaged group did not differ from that of the discharged group in terms of the demographic variables and other diagnosis and service information. The only exceptions were that patients in the disengaged group attended fewer therapy sessions and had more missed appointments. A high number of missed appointments could suggest that these patients had difficulty committing to weekly sessions, not that they decided to disengage from therapy one day. In unpublished data collected for the same service, the disengaged clients were contacted and asked about their reasons for not showing up to their appointments. Eighty per cent of the sample reported that transportation difficulties were the primary cause of missing appointments. In this unpublished survey, none of the patients in the sample reported reasons related to the services provided or negative outcomes. This finding could provide assurance that our disengagement rate might not be related to the ineffectiveness of the treatment intervention. 
However, this should be confirmed with future studies designed around a follow-up and aiming to understand when and why clients disengage from therapy.

Different means of service delivery, for example online therapy or various applications, are also recommended to tackle attendance difficulties. T-CBT can work well in such programmes because it is flexible enough to work with various forms of difficulties and does not require prior lengthy diagnostic assessment. However, the challenge would be in designing an online protocol that allows the flexibility for making decisions as to which elements should be proposed for the current difficulties rather than just providing a fixed module to all clients. Much research and service development is needed to tackle not only transportation difficulties but also access to clients in remote areas where psychological services might not be available.

Additionally, in many CBT trials and protocols, the average number of sessions is between 12 and 16, e.g. Barlow et al. (2017) and Norton (2012b). In our study, the protocol was flexible regarding the number of sessions each patient should receive; it was suggested that after 16 sessions, the case should be reviewed to determine difficulties and a possible resolution. During the study period, there were only two patients who reached this number of sessions and required such discussion and resolution. The average number of sessions the patients received was 5 ( $S D$ 4.7) in 3.5 months ( $S D$ 4.5); the patients who completed the programme had a higher number of sessions $(9.1 ; S D$ 6.3) over a longer period of time (6.2 months; SD 5.4), whereas the clients in the HI group received more sessions over a longer period. However, the average number of session in all the groups (5-9 sessions) remained relatively short compared with that of existing protocols and CBT trials. In our view, this is one of the important findings of this study when considering the potential cost-effectiveness of T-CBT. Our flexible design allows us to test the number of sessions needed for each client individually rather than just using a fixed pre-determined number of sessions. This again emphasizes the patient's perspective and needs when designing intervention programmes because it could lead to better outcomes and lower costs, as each client receives what he/she needs (no less, no more).

One major aim of this study was to assess the applicability of this LI intervention delivered by junior psychologists. The result of the study confirms the feasibility of this approach and confirms that T-CBT is effective in reducing various levels of symptoms reported by clients and as judged by clinicians. This has important clinical implications especially in countries such as Saudi Arabia that have a large number of junior psychologists who can be a potential good source of psychotherapy workforce, thereby reducing the treatment gap in mental health services. It is recommended that such programmes be implemented in suitable settings where at least one senior supervisor psychologist implements the task sharing model with other junior staff members; however, such implementation requires regular progress monitoring and outcome evaluation to confirm these initial findings.

To summarize, this study provides initial yet important evidence for the applicability of T-CBT in Saudi Arabia, especially when it is delivered by junior psychologists as a LI. The results suggest a number of implications for clinical services and training opportunities as well as generating more questions for future research to tackle. However, this study is not without limitations, one of which is the small sample size of the group who completed the programme. Other limitations include the lack of information about patient feedback on therapy and the reasons why some patients chose to disengage from treatment. Thus, future research should aim to replicate these findings and tackle these important issues.

Author ORCID. Yousra Alatiq https://orcid.org/0000-0002-1562-6693

Acknowledgements. We would like to thank all the psychologists involved in this study for their hard work and commitment in delivering the treatment protocol and collecting the data.

Financial support. There was no financial support for this study. 
Conflicts of interest. The authors declare no conflicts of interest.

Ethical statement. The authors have abided by the Ethical Principles of Psychologists and Code of Conduct as set out by the APA. The study was reviewed and approved by the IRB at King Abdullah International Medical Research Centre (KAIMRC), KSA.

Key practice points

(1) T-CBT provides the potential for improving psychotherapy services in countries with limited resources.

(2) There is initial evidence that T-CBT is suitable for clients with emotional disorders in Saudi Arabia.

(3) There is initial support for the effect of T-CBT as a low-intensity intervention delivered by junior psychologists.

\section{References}

Alatiq, Y. (2014). Transdiagnostic cognitive behavioural therapy (CBT): case reports from Saudi Arabia. Cognitive Behaviour Therapist, 7, e2.

Alqahtani, M. M. J. (2019). Unpublished results presented at the meeting for the national plan to improve the practice of psychologists and psychotherapy. Saudi Health Council. Riyadh, Saudi Arabia.

Albuhairi, A. A. (2005). SCL-90-R Arabic version. Asuod, Eyget: Counseling \& Educational Center.

Andersen, P., Toner, P., Bland, M., \& McMillan, D. (2016). Effectiveness of transdiagnostic cognitive behaviour therapy for anxiety and depression in adults: a systematic review and meta-analysis. Behavioural and Cognitive Psychotherapy, 44, 673-690.

Barlow, D. H., Allen, L. B., \& Choate, M. L. (2004). Toward a unified treatment for emotional disorders. Behavior Therapy, 35, 205-230.

Barlow, D. H., Farchione, T. J., Bullis, J. R., Gallagher, M. W., Murray-Latin, H., Sauer-Zavala, S., \& Ametaj, A. (2017). The unified protocol for transdiagnostic treatment of emotional disorders compared with diagnosis-specific protocols for anxiety disorders: a randomized clinical trial. JAMA Psychiatry, 74, 884-875.

Barlow, D. H., Farchione, T. J., Sauer-Zavala, S., Latin, H. M., Ellard, K. K., Bullis, J. R., Bentley, K. H., Boettcher, H. T., \& Cassiello-Robbins, C. (2011). Unified Protocol for Transdiagnostic Treatment of Emotional Disorders: Therapist Guide. New York, USA: Oxford University Press.

Brown, T., \& Barlow, D. (2002). Classification of anxiety and mood disorders. Anxiety and its Disorders: the Nature and Treatment of Anxiety and Panic, 2, 292-327.

Bullis, J. R., Sauer-Zavala, S., Bentley, K. H., Thompson-Hollands, J., Carl, J. R., \& Barlow, D. H. (2015). The unified protocol for transdiagnostic treatment of emotional disorders: preliminary exploration of effectiveness for group delivery. Behavior Modification, 39, 295-321.

Carrey, T. A. (2016). Beyond patient-centred care: enhancing the patient experience in mental health services through patient-perspective care. Patient Experience Journal, 3, 2.

Clark, D. A., \& Taylor, S. (2009). The transdiagnostic perspective on cognitive-behavioral therapy for anxiety and depression: new wine for old wineskins? Journal of Cognitive Psychotherapy, 23, 60-66.

Derogatis, L. R. (1977) SCL-90-R Administration, Scoring and Procedures Manual. Baltimore, MD, USA: Clinical Psychometric Research.

Fairburn, C. G., Cooper, Z., \& Shafran, R. (2003). Cognitive behaviour therapy for eating disorders: a 'transdiagnostic' theory and treatment. Behaviour Research and Therapy, 41, 509-528.

Ferrans, C. E., \& Powers, M. J. (1985). Quality of life index: development and psychometric properties. Advances in Nursing Science, 8, 15-24.

García-Escalera, J., Chorot, P., Valiente, R. M., Reales, J. M., \& Sandín, B. (2017). Eficacia de la terapia cognitivo conductual transdiagnóstica en el tratamiento de la ansiedad y la depresión en adultos, niños y adolescentes: un meta-análisis. Revista de Psicopatología y Psicología Clínica, 21, 147-175.

Guy, W. (1976). Clinical Global Impressions. ECDEU Assessment Manual for Psychopharmacology. Rockville, MD, USA: US Department of Health, Education, and Welfare, Public Health Service, Alcohol, Drug Abuse, and Mental Health Administration, National Institute of Mental Health, Psychopharmacology Research Branch, Division of Extramural Research Programs.

Harvey, A., Watkins, E., Mansell, W., \& Shafran, R. (2004) Cognitive Behavioural Processes Across Psychological Disorders: A Transdiagnostic Approach to Research and Treatment. USA: Oxford University Press.

Kroenke, K., Spitzer, R. L., \& Williams, J. B. W. (2001). The PHQ-9: validity of a brief depression severity measure. Journal of General Internal Medicine, 16, 606-613. 
Murray, L. K., Dorsey, S., Bolton, P., Jordans, M. J., Rahman, A., Bass, J., \& Verdeli, H. (2011). Building capacity in mental health interventions in low resources countries: an apprenticeship model for training local providers. International Journal of Mental Health Systems, 5, 30.

Murray, L. K., Dorsey, S., Haroz, E., Lee, C., Alsiary, M. M., Haydary, A., \& Bolton, P. (2014). A common elements treatment approach for adult mental health problems in low-and middle-income countries. Cognitive and Behavioral Practice, 21, 111-123.

Newby, J. M., McKinnon, A., Kuyken, W., Gilbody, S., \& Dalgleish, T. (2015). Systematic review and meta-analysis of transdiagnostic psychological treatment for anxiety and depressive disorders in adulthood. Clinical Psychology Review, 40, 91-110.

Norton, P. J. (2012a). Group Cognitive-Behavioral Therapy of Anxiety: A Transdiagnostic Treatment Manual. New York, NY, USA: Guilford Press.

Norton, P. J. (2012b). Transdiagnostic group CBT for anxiety disorder: efficacy, acceptability, and beyond. Spanish Journal of Clinical Psychology, 17, 205-217.

Padesky, C. A., \& Mooney, K. A. (1990). Presenting the cognitive model to clients. International Cognitive Therapy Newsletter, 6, 13-14.

Sheehan, V. (1983). Sheehan Disability Scale- overview. Available at: http://memorialparkpsychiatry.com/doc/sheehan_ disability_scale.pdf

Spitzer, R. L., Kroenke, K., Williams, J. B.W., \& Löwe, B. (2006). A brief measure for assessing generalized anxiety disorder: the GAD-7. Archives of Internal Medicine, 166, 1092-1097.

Young, J., \& Beck, A. T. (1980). Cognitive therapy scale: rating manual. Unpublished manuscript.

Cite this article: Alatiq Y and Al Modayfer O. Transdiagnostic CBT for adult emotional disorders: A feasibility open trial from Saudi Arabia. The Cognitive Behaviour Therapist. https://doi.org/10.1017/S1754470X19000163 